\title{
Are Sports Betting Markets Semistrong Efficient? Evidence from the Covid-19 Pandemic
}

Meier, Pascal Flurin ; Flepp, Raphael ; Franck, E

\begin{abstract}
This paper examines whether sports betting markets are semi-strong form efficient-i.e., whether new information is rapidly and completely incorporated into betting prices. We use news on ghost games in the top European football leagues due to the COVID-19 pandemic as a clean arrival of new public information. Because spectators are absent during ghost games, the home advantage is reduced, and we test whether this information is fully reflected in betting prices. Our results show that bookmakers and betting exchanges systematically overestimated a home team's winning probability during the first period of the ghost games, which suggests that betting markets are, at least temporally, not semi-strong form efficient. Examining different leagues, we find that our main results are driven by the German Bundesliga, which was the first league to resume operations. We exploit a betting strategy that yields a positive net payoff over more than one month.
\end{abstract}

DOI: https://doi.org/10.32731/IJSF/163.082021.01

Posted at the Zurich Open Repository and Archive, University of Zurich

ZORA URL: https://doi.org/10.5167/uzh-206447

Journal Article

Published Version

Originally published at:

Meier, Pascal Flurin; Flepp, Raphael; Franck, E (2021). Are Sports Betting Markets Semistrong Efficient? Evidence from the Covid-19 Pandemic. International Journal of Sport Finance, 16(3):111-126.

DOI: https://doi.org/10.32731/IJSF/163.082021.01 


\title{
Are Sports Betting Markets Semi-Strong Efficient? Evidence From the COVID-19 Pandemic ${ }^{1}$
}

\author{
Pascal Flurin Meier, Raphael Flepp, and Egon Franck \\ University of Zurich
}

Pascal Flurin Meier, is a student in the Department of Business Administration at the University of Zurich. His research interests include behavioral economics and corporate governance.

Raphael Flepp, PhD, is a senior research and teaching associate in the Department of Business Administration at the University of Zurich. His research interests include economics of strategy and behavioral economics.

Egon Franck, PhD, is a professor of strategic management and business policy in the Department of Business Administration at the University of Zurich. His research interests include economics of strategy, governance structures, and incentive mechanisms.

\begin{abstract}
This paper examines whether sports betting markets are semi-strong form efficient-i.e., whether new information is rapidly and completely incorporated into betting prices. We use news on ghost games in the top European football leagues due to the COVID-19 pandemic as a clean arrival of new public information. Because spectators are absent during ghost games, the home advantage is reduced, and we test whether this information is fully reflected in betting prices. Our results show that bookmakers and betting exchanges systematically overestimated a home team's winning probability during the first period of the ghost games, which suggests that betting markets are, at least temporally, not semi-strong form efficient. Examining different leagues, we find that our main results are driven by the German Bundesliga, which was the first league to resume operations. We exploit a betting strategy that yields a positive net payoff over more than one month.
\end{abstract}

Keywords: sports betting market, market efficiency, home advantage, COVID-19

http://doi.org/10.32731/IJSF/163.082021.01

\section{Introduction}

There has been an ongoing debate in the empirical literature about the extent to which information markets are efficient. Drawing from the well-established framework developed by Eugen Fama in 1970, market efficiency may be categorized into weak, semi-strong, or strong forms depending on the amount of information that is reflected in prices. Empirical studies have increasingly utilized betting markets to assess the information efficiency of markets. Such markets offer economists an ideal setting in which to examine how information efficiency operates compared to traditional stock markets, which, due to their structural characteristics, have some serious drawbacks (Croxson \& Reade, 2014). From an economic perspective, betting markets, which have already become larger than their underlying sports markets according to the Fédération Internationale de Football Association (FIFA, 2011), are crucial and continue to surge in popularity with the digital revolution in full swing.

Despite the increase in research on betting markets, evidence regarding the degree to which these markets are efficient is mixed, as Angelini and De Angelis (2019) highlighted. The bulk of studies on information efficiency focus on the weak form of information efficiency, while evidence of the semi-strong form is scarce (Bernardo et al., 2019). A major obstacle remains in terms of cleanly assessing the arrival of new public information in semi-strong market efficiency studies. In this paper, we are able to tackle this issue by using a natural experiment. In early 2020, the outbreak of COVID-19 suddenly brought the sports world and public life in general to a standstill for several months. It was not surprising that banning large sports events, which are potential epicenters of infection, was one of the first preventive measures of governments to restrict the further spreading of the virus. The situation in Europe eased over the course of the next few months, which allowed soccer-which we subsequently refer to as football-leagues to gradually resume operations. However, none of the top European football leagues allowed spectators in their stadiums, at least for the end of the 2019/2020 season. 
Most spectators at a given game are supporters of the home team, which contributes to an increased probability of the home team winning, an effect called the home advantage (Goumas, 2014; Nevill et al., 1996; Pollard, 2006; Ponzo \& Scoppa, 2018). News of "ghost games" can be considered a clean arrival of new public information: The decision to host spectator-less games was announced after the hygiene procedures were approved by the respective governments but well before the league games resumed. Moreover, the information that teams have to play in empty stadiums is highly relevant because the absence of supporters substantially reduces the home advantage (Pettersson-Lidbom \& Priks, 2010; Ponzo \& Scoppa, 2018). As such, COVID-19-induced ghost games provide an ideal setting in which to properly analyze the hypothesis of semi-strong market efficiency in European professional football; we can use this case to examine the extent to which the arrival of new public information, i.e., ghost games without spectators and thus a reduced home advantage, is reflected in betting markets.

We investigate football games played from the 2014/2015 season to the 2019/2020 season. In particular, we analyze 8,676 games in the most prominent European football leagues in Italy, Germany, England, and Spain. ${ }^{2}$ We find that while bookmaker odds and closing odds from betting exchanges were unbiased prior to the lockdown, the betting market systematically overestimated the home team's chances of winning during COVID-19-induced ghost games. Our results suggest that betting markets are not efficient, at least not in a semi-strong form. However, we also find that this inefficiency gradually decreases over time. The average effect of ghost games on home advantage is incorrectly incorporated into betting odds for five weeks after the German Bundesliga resumed operations as the first major European football league. Examining the leagues separately, we find that our main results are driven by the German Bundesliga. The fact that both market structures are, at most, weak-form efficient indicates that they provide ample opportunities for investors to earn abnormal returns by quickly reacting to surprise announcements and disclosures. Indeed, we document a large positive cumulative betting return for the first weeks after resumption of the German Bundesliga achieved through a simple betting strategy.

We contribute to the existing literature in several ways. First, we examine market efficiency in the context of betting markets, which offer considerable advantages over conventional financial markets (Croxson \& Reade, 2014). Second, by utilizing COVID-19-induced ghost games, we exploit a research design in which new public information about the relative playing strength of home and away teams arrives in the market cleanly. In contrast to studies utilizing in-play betting data (Choi \& Hui, 2014; Croxson \& Reade, 2014), our pre-play betting setting does not suffer from price drift issues. Third, the empirical evidence on semi-strong-form efficiency in betting markets is mixed (Bernardo et al., 2019). Thus, our findings complement a still inconclusive strand of research about semi-strong market efficiency in betting markets. Fourth, we examine the efficiency for both bookmakers and betting exchanges jointly, while existing studies on semi-strong betting market efficiency focus on either bookmakers (Bernardo et al., 2019; Deutscher et al., 2018; Fischer \& Haucap, 2020a; Hegarty, 2021; Winkelmann et al., 2021) or betting exchanges (Choi \& Hui, 2014; Croxson \& Reade, 2014).

In contemporaneous work, Winkelmann et al. (2021) and Fischer and Haucap (2020a) examined a similar research question for ghost games played in Germany. In addition to these studies, we analyze the four major European football leagues to exploit the staggered resumption of league operations. To the best of our knowledge, Hegarty (2021) is the only contemporaneous study that also examined the major European leagues, but the author utilizes the Asian handicap market.

The remainder of this paper is structured as follows. The related literature is discussed in the second section. In the third section, we discuss the effect of ghost games on home advantage and present the research design and the data utilized. In the fourth section, we carry out an empirical analysis and present the results. The fifth section concludes the paper with a discussion of the results.

\section{Semi-Strong Form Market Efficiency in Betting Markets}

The question of how markets incorporate the arrival of new information has long been of interest to both practitioners and academics. Given its importance for investment opportunities and investor roles, the question of market efficiency has attracted abundant empirical research. However, due to the structural characteristics of conventional financial markets, some serious drawbacks complicate a clean test of market efficiency hypotheses (Croxson \& Reade, 2014). On the one hand, it is difficult to determine when news is absorbed by the market and to rule out information leakage. On the other hand, the definition of a normal return remains obscure because the fundamental values of traditional financial products 
are not observable. Therefore, equilibrium models become necessary to define a normal security return. Such studies suffer from the joint hypothesis problem: Rejecting market efficiency may be due to a real inefficiency in the price mechanism or because the equilibrium model is incorrect (Borghesi, 2014; Croxson \& Reade, 2014; Franke, 2020; Marshall, 2009).

To allow for a cleaner test of the market efficiency hypothesis, a growing strand of literature switched to a rather unconventional financial market: the sports betting market. Vaughan Williams (2005) provided an introduction to information efficiency in betting markets. This market offers some unique features that create a sort of "laboratory setting" for studying market efficiency (Hvattum, 2013): A large number of experienced investors (bettors) with access to information and assets (betting contracts) are acting in a real market. Not only does a betting contract have a clear endpoint at which its true value is revealed, but also its outcome (for instance, a draw between a home and an away team) is not affected by macroeconomic factors (for instance, international trade conflicts) or bettor expectations (Flepp et al., 2017). Hence, betting markets may offer a superior lens for efficiency studies, particularly in the case of the clean arrival of new information (Croxson \& Reade, 2014).

Most of the empirical work around inefficiencies in betting markets is centered around studies investigating the weak form of market efficiency in sports betting (Angelini \& De Angelis, 2019; Dixon \& Coles, 1997; Dixon \& Pope, 2004; Goddard \& Asimakopoulos, 2004; Kuypers, 2000; Marshall, 2009; Rue \& Salvesen, 2000; Vlastakis et al., 2009; Winkelmann et al., 2020). Winkelmann et al. (2020) determined that most of the strategies exploiting biases in betting odds do not generate positive returns in the long run but may be caused by chance and statistical noise. Even if certain biases exist in betting odds, another question is whether such strategies generate abnormal returns after the consideration of transaction costs such as the bookmaker's commission. For example, Berkowitz, Depken II, and Gandar (2017) found that a betting strategy that exploits a favorite-longshot bias in the fixed-odds betting market from U.S. college basketball and football leads to an average return of near zero, which is larger than the return of a random bet, and thus to the elimination of the bookmaker's commission. However, as no positive returns can be generated, the authors concluded that these markets are efficient within transaction costs.

Bernardo et al. (2019) highlighted that in contrast to the weak form of market efficiency, the semi-strong form is less extensively analyzed in the literature. Predominantly, studies on semi-strong form tests are conducted in the context of horse race betting (Sung et al., 2005). However, the results of these studies are mixed and inconclusive (Cain et al., 2000; Edelman, 2002; Hausch et al., 1981; Smith, 2002; Sung et al., 2005). With respect to football, Elaad et al. (2020) tested for a version of semi-strong efficiency, i.e., whether there is an opportunity for bettors to achieve better returns without losing the bookmaker's profit margin. The authors found no statistically significant evidence that the overall market is inefficient. Another strand of research examines the effect of experts' predictions in the main national media, as Bernardo et al. (2019) noted. Despite expert predictions being more accurate than chance, Forrest and Simmons (2000) highlighted that the individual expertise that tipsters can claim to offer is limited; they fail to properly incorporate publicly available information.

A semi-strong efficient market requires prices to immediately reflect new information once it becomes public knowledge. Indeed, Croxson and Reade (2014) showed with data from a betting exchange that prices update swiftly following a scored goal in football, indicating that betting markets seem to incorporate market news rapidly and completely. By contrast, Choi and Hui (2014) rejected the hypothesis of semi-strong market efficiency using similar live football betting data: They found that prices generally underreact to normal news and overreact to surprising news. Bizzozero et al. (2018) suggested that fast traders promote quick price discovery and correctly incorporate new information into prices, examining courtside trading during live tennis. Brown et al. (2018) found that social media content contains additional predictive power not included in betting prices. In particular, after significant market events, such as goals and red cards, Twitter activity may help in the interpretation of information.

For pre-play betting markets, Bernardo et al. (2019) found evidence of semi-strong form inefficiency utilizing a major change in expectation about team results, i.e., when the head coach of a team is replaced. The authors found that a betting strategy that makes bettors place a proportional stake on the victory of the team that changed the coach yields a positive return for the first four games after replacement. Deutscher et al. (2018) found evidence of temporal market inefficiencies when betting on recently promoted teams, which typically undergo major changes in the composition of the roster following promotion. Market inefficiencies remain only at the start of the season since bookmakers have difficulty 
predicting the playing strength of recently promoted teams at first. Thus, bookmakers may lack reliable information during certain periods, which leads to betting market inefficiencies.

In contemporaneous work, Winkelmann et al. (2021) and Fischer and Haucap (2020a) showed that bookmakers misprice the home advantage during the ghost games played in Germany. Winkelmann et al. (2021) found that the misassessment persists until the end of the season, and the results of Fischer and Haucap (2020a) suggested a slow to non-existing adaption process over time. In a related study, Hegarty (2021) examined the efficiency of the Asian handicap football betting market for each of the four major European leagues. In contrast, the author found that the bookmaker market becomes more efficient during ghost games. Hegarty (2021) suggested that since the crowd component is subjective and difficult to quantify, the removal leads to improved prediction accuracy.

\section{Research Design}

\section{Spectators and the Home Advantage}

The phenomenon of home advantage-a term referring to the finding that home teams in sports competitions win more of the games played under a balanced home and away schedule (Courneya \& Carron, 1992) - is very well documented in competitive sports. ${ }^{3}$ There exist several underlying mechanisms, including crowd support, referee bias, territoriality effects, travel effects, and familiarity effects (Van Damme \& Baert, 2019). ${ }^{4}$

If crowd support is vital for success when playing in the home venue, COVID-19-induced ghost games are expected to significantly alter the home advantage. Indeed, studies suggest that the COVID-19 pandemic has significantly affected the home advantage (Benz \& Lopez, 2020; Bryson et al., 2021; Cueva, 2020; Dilger \& Vischer, 2020; Endrich \& Gesche, 2020; Ferraresi \& Gucciardi, 2020; Fischer \& Haucap, 2020b; Konaka, 2021; Leitner \& Richlan, 2020; McCarrick et al., 2020; Reade \& Singleton, 2020; Scoppa, 2021; Sors et al., 2020; Tilp \& Thaller, 2020).

Examining 41 professional football leagues, Cueva (2020) suggested that the home advantage drops by approximately half on average and that the referee bias against the away team vanishes completely during ghost games. Scoppa (2021) documented that while home teams score approximately 0.50 points more with a crowd, the advantage in points is reduced to approximately 0.28 in ghost games. Similarly, the difference in goals is halved in games without spectators. Ferraresi and Gucciardi (2020), utilizing five top European football leagues, found that home team performance is halved when stadiums are empty. The effect is more marked for teams that are used to a full crowd and have international experience. Fischer and Haucap (2020b) found a reduced home advantage in the Bundesliga but not in lower divisions, arguing that these teams are potentially more used to playing in half-empty stadiums. ${ }^{5}$ Bryson et al. (2021, p. 3) graphically illustrated the change in home advantage within professional football leagues. Based on goal and yellow card differences, most of the leagues, including the major four European leagues, exhibit a decreased home advantage.

Well before the pandemic, Ponzo and Scoppa (2018) found a sizable effect of crowd support on home advantage utilizing same-stadium derbies. This finding is consistent with Pettersson-Lidbom and Priks (2010), who examined Italian football games that were played in empty stadiums due to hooligan violence. Not only among researchers but also among fans, the general public, and thus presumably bettors, the phenomenon of home advantage is well known. Even before the German Bundesliga resumed operations, and particularly after the first game day, articles drew attention to the disappearance of, or at least the reduction in, the home advantage. ${ }^{6}$

Thus, the pandemic provides a clean setting of the home advantage being exogenously altered and new public information arriving in the market. An efficient betting market, at least in semi-strong form, requires that betting odds fully incorporate new public information once it is released. Thus, the winning probability of home teams should be smaller in ghost games compared to non-ghost games, while the winning probability of away teams should increase. We analyze the extent to which the betting markets have incorporated this change in the magnitude of the home advantage and may thus be perceived as efficient.

\section{Method and Data}

We focus on the most prominent European leagues. Thus, our dataset features the first divisions of the following countries: England, Germany, Italy, and Spain. We expect that the incentives to accurately price betting odds are largest for 
these leagues since they attract the most attention. We exclude France, as the French League 1 schedule was terminated without playing ghost games.

Depending on the league, 9 to 12 match rounds were played behind closed gates. The leagues resumed operations at different points in time. The German Bundesliga was the first league to restart, with ghost games in mid-May. The Spanish Primera Division, the second top league to resume operations, restared almost one month later; the English Premier League was next, and Italy's Serie A was last. The timeline in Figure 1 shows the developments in the top five European football leagues in 2019/2020.

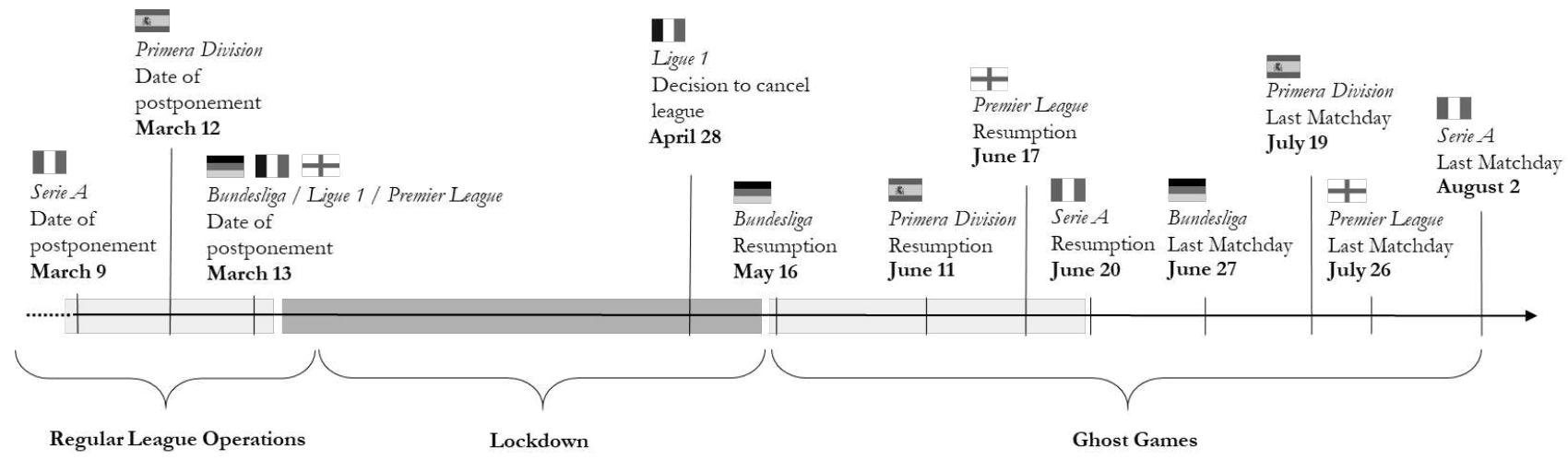

Figure 1. Timeline.

Notes: The figure displays the developments and decisions in the top five European football leagues with respect to the COVID-19 pandemic.

Betting markets may be categorized into two different forms (Franck et al., 2010): the bookmaker market, where bookmakers act as dealers and provide odds against which bettors can place bets (the quote-driven market), and betting exchanges, where prices are determined by bettors trading the bets among themselves (the order-driven market).

We collected bookmaker data from www.football-data.co.uk, a website delivering comprehensive football statistics and betting odds from leagues around the world. The data include the odds of several bookmakers, such as Bet365, Betwin, Interwetten, Pinnacle, William Hill, and VC Bet. Data for two major betting exchange platforms, Matchbook and Betfair, were provided by www.indatabet.com. We collected data for five seasons spanning from the 2014/2015 season to the 2019/2020 season, resulting in a total of 8,676 individual games.

The reciprocals of the odds represent the estimation of the probability of a win, defeat, or draw. In a frictionless market, the probabilities should sum to 1 . In practice, however, they rarely do so due to transaction costs and bookmakers' margins on all odds to cover the uncertainty of a game's outcome, the so-called overround (Bizzozero et al., 2016). To account for this, we derive the implied probabilities for all possible game outcomes, i.e., a win of the home team $\left(\mathrm{O}_{h}\right)$, draw $\left(\mathrm{O}_{d}\right)$, or a win of the away team $\left(\mathrm{O}_{a}\right)$ (Deutscher et al., 2018). ${ }^{7}$ We derive the implied probabilities for the six bookmakers-Bet365, Betwin, Interwetten, Pinnacle, William Hill, and VC Bet-and then calculate the average book probability of the bookmakers (BookProb). The average implied probability for the two betting exchanges (ExchangeProb) is calculated in the same way. ${ }^{8}$ In Table 1, descriptive statistics of the variables are displayed.

For the main analysis of this paper, we test the market efficiency of betting markets by means of regression methods to examine whether the implied winning probabilities of the home and away team alone are sufficient to predict the game outcome. Thus, we regress the outcome of the game, which is equal to one if the team under consideration won and zero otherwise, on the implied probability (ImpWinProb), equaling either BookProb (bookmaker sample) or ExchangeProb (betting exchange sample), of a home team win or away team win and additional covariates. If betting markets are efficient and incorporate new information correctly, only the implied probabilities should predict the game outcome (Bizzozero et al., 2018). Under this scenario, we would expect the effects of covariates to be close to zero. By contrast, if 
Table 1 Descriptive Statistics

\begin{tabular}{lcccccc}
\hline & $\mathrm{N}$ & Mean & Q1 & Median & Q3 & SD \\
\hline $\begin{array}{l}\text { Variables Used in the Regression } \\
\text { (Home and Away Perspectives) }\end{array}$ & & & & & & \\
Outcome & 17,352 & 0.377 & 0.000 & 0.000 & 1.000 & 0.485 \\
BookProb & 17,352 & 0.376 & 0.226 & 0.354 & 0.505 & 0.195 \\
ExchangeProb & 17,352 & 0.377 & 0.219 & 0.354 & 0.512 & 0.204 \\
Time & 17,352 & 0.310 & 0.000 & 0.000 & 0.000 & 1.504 \\
GhostGame & 17,352 & 0.047 & 0.000 & 0.000 & 1.000 & 0.212 \\
Home & 17,352 & 0.500 & 0.000 & 0.500 & 1.000 & 0.500 \\
\hline
\end{tabular}

Notes: This table reports descriptive statistics for the variables employed and additional information at the game level.

the coefficients of the covariates provide additional explanatory power above and beyond the implied probabilities, we would conclude that betting markets are inefficient. Thus, small $p$-values of the covariates provide statistical evidence that implied probabilities do not accurately reflect the conditional probability of the game outcome (Choi \& Hui, 2014).

To capture a potential general home bias in the odds, we include the variable Home, indicating whether the team is playing at home or away. We further include the variable GhostGame, indicating games played after the leagues resumed operations: The variable equals 1 for all games played after May 15, 2020. We include an interaction term Home x GhostGame. If markets digest all relevant information correctly, the covariates should not yield any explanatory power. As our response variable is binary, equaling 1 for a win and 0 for a defeat, we estimate the following logit model:

$$
\begin{gathered}
\mathrm{Y}_{\mathrm{i}, \mathrm{m}}=\beta_{0}+\beta_{1} \text { ImpWinProb }_{\mathrm{i}, \mathrm{m}}+\beta_{2} \text { Home }_{\mathrm{i}, \mathrm{m}}+\beta_{3} \text { GhostGame }_{\mathrm{m}}+\beta_{4} \text { Home }_{\mathrm{i}} \mathrm{x} \\
\text { GhostGame }_{\mathrm{m}}+\alpha_{\mathrm{j}} \\
\mathrm{f}(\pi)=\log \left(\frac{\pi_{\mathrm{i}, \mathrm{m}}}{1-\pi_{\mathrm{i}, \mathrm{m}}}\right)=\mathrm{Y}_{\mathrm{i}, \mathrm{m}}
\end{gathered}
$$

where $i$ denotes the home or away team and $m$ denotes the specific game. The probability $\pi$ is combined with the linear predictor $Y$ through the logit link function (Deutscher et al., 2018). We follow the established literature (Deutscher et al., 2018; Forrest \& Simmons, 2008) by looking at the games from the perspective of both the home and the away team. Thus, for every game, we have two observations: betting odds on the win of the home team and betting odds on the win of the away team. ${ }^{9}$ Further, we include fixed effects $\alpha_{j}$ for each league $j .{ }^{10}$ Standard errors are clustered at the game level to account for pairwise correlation. We are mainly interested in $\beta_{4}$, which indicates market inefficiency during COVID-19induced ghost games.

In further analysis, we include the variable Time, indicating the number of weeks since the first resumption day to account for whether market inefficiency persists over time or diminishes. ${ }^{11}$ Thus, we interact Home with Time, which extends Equation 1 to the following form:

$$
\begin{aligned}
& Y_{i, m}=\beta_{0}+\beta_{1} \text { ImpWinProb }_{i, m}+\beta_{2} \text { Home }_{i, m}+\beta_{3} \text { GhostGame }_{m}+\beta_{4} \text { Time }_{m}+\beta_{5} \text { Home }_{i} x \\
& \text { GhostGame }_{m}+\beta_{6} \text { Home }_{i} \text { Time }_{m}+\alpha_{j}
\end{aligned}
$$

We are mainly interested in the coefficient $\beta_{5}$, which indicates the average effect of a home bias during the ghost games, and in the coefficient $\beta_{6}$, which indicates whether any such bias increases or decreases over time. 


\section{Results}

For the main analysis, we estimate Equation 1. Table 2 shows the baseline estimations of market efficiency during the COVID-19 crisis. We estimate Equation 1 with different samples that vary with respect to the time period employed. All samples include games from 2014/2015 until the lockdown in early 2020. Additionally, they include ghost games starting from the resumption date in the German Bundesliga on Saturday, May 16, 2020, until the date specified. We start with a sample period until Sunday, May 24, 2020, i.e., eight days including two weekends of ghost games. Subsequently, we increase the time period of ghost games in weekly steps to exploit the staggered resumption of the major European leagues. From June 28, 2020, onwards (all leagues resumed by June 20,2020), we show estimations using a larger interval until the last ghost game of the 2019/2020 season played in European top football leagues (August 2, 2020). ${ }^{12}$ In Panel A of Table 2, we show the estimations utilizing bookmaker probabilities, while in Panel B of Table 2, we utilize the average implied probabilities derived from the betting exchanges. ${ }^{13}$

The results from Panel A of Table 2 suggest that, on average, there is no home bias by bookmakers, i.e., higher odds compared to the fair odds prior to the lockdown, as the coefficient of Home is not statistically significant. ${ }^{14}$ As expected, the average book probabilities are a highly significant predictor of the game outcome. However, the estimates also show a home bias during the early COVID-19-induced ghost games, which is indicated by the significantly negative interaction term of Home x GhostGame. Statistical significance is present for the sample period until June 21, 2020 for both market structures. The winning probabilities are significantly overestimated for the home team during ghost games, as suggested by the negative coefficient of the interaction term. Thus, the implied winning probability of the home (away) team as predicted by the bookmakers is higher (lower) than the actual occurrence of a win. We derive the same conclusions if we focus on the implied probabilities derived from betting exchange odds in Panel B of Table 2. ${ }^{15}$

The results indicate that the implied winning probability of the home (away) team as predicted is higher (lower) than the actual occurrence of a win. This suggests that new market information is not immediately priced into betting odds: Bookmakers and bettors did not accurately predict the COVID-19-induced ghost games and their impact on the home and away teams' winning probabilities. The results remain virtually the same when we change the estimation model (OLS, probit) or employ the odds of the betting exchanges or bookmakers individually. ${ }^{16}$

The results in Table 2 also show that the inefficiency was largest during the first days after resumption, whereas it gradually decreased and vanished toward the end. There, findings suggest that market inefficiencies are temporal and that bookmaker odds, on average, did not fully reflect the impact of ghost games on home advantage for five weeks after resumption in the German Bundesliga. The same applies for the closing betting odds quoted in the betting exchange, showing a very similar pattern.

To investigate the time trend more systematically, we estimate Equation 3. The results in Table 3 indicate that the implied winning probability is again a highly significant predictor of actual game outcome. As expected, Time is not statistically significant. On average, there was no home bias prior to the lockdown period, as indicated by Home. However, the interaction term of Home $\mathrm{x}$ GhostGame is statistically significant at the $1 \%$ level, suggesting that the betting market mispriced the home advantage during ghost games. Bookmakers and bettors alike systematically overestimated the probability of winning when playing at home, as indicated by the negative coefficient of Home x GhostGame. However, this effect vanished over time, as shown by the positive and statistically significant coefficient of Home $\mathrm{x}$ Time.

To investigate the incorporation of new information into the betting odds further, we subsequently split our sample and analyze each league separately. The staggered resumption of league operations allows us to examine information processing more deeply. We would expect to see the strongest effect in the German Bundesliga-which resumed operations almost one month prior to the next league in our sample.

Indeed, the results in Table 4 suggest that our main results are mostly driven by the German Bundesliga. We see no evidence that bookmakers misprice the home advantage in the other three leagues, which also holds when examining pricing over time. ${ }^{17}$ Thus, bookmaker odds fully reflected the impact of ghost games on home advantage in these leagues, even immediately after resuming operations. The same applies for the closing betting odds quoted in the betting exchanges. 
Table 2. Baseline Estimation

\begin{tabular}{|c|c|c|c|c|c|c|c|c|}
\hline \multirow[b]{2}{*}{ Panel A: Bookmaker } & \multicolumn{8}{|c|}{ Outcome } \\
\hline & $\begin{array}{c}\text { I } \\
+8 \mathrm{~d} . \\
\text { May } 24\end{array}$ & $\begin{array}{c}\text { II } \\
+15 \mathrm{~d} . \\
\text { May } 31\end{array}$ & $\begin{array}{c}\text { III } \\
+22 \mathrm{~d} . \\
\text { June } 7\end{array}$ & $\begin{array}{c}\text { IV } \\
+29 \mathrm{~d} . \\
\text { June } 14\end{array}$ & $\begin{array}{c}\mathrm{V} \\
+36 \mathrm{~d} \text {. } \\
\text { June } 21\end{array}$ & $\begin{array}{c}\text { VI } \\
+43 \mathrm{~d} . \\
\text { June } 28\end{array}$ & $\begin{array}{c}\text { VII } \\
+64 d . \\
\text { July } 19\end{array}$ & $\begin{array}{l}\text { VIII } \\
+78 \text { d. } \\
\text { Aug. } 2\end{array}$ \\
\hline \multirow{2}{*}{ Constant } & $-2.489^{\star * *}$ & $-2.490^{\star * *}$ & $-2.489^{\star * *}$ & $-2.491^{\star * *}$ & $-2.490^{* * *}$ & $-2.498^{\star * *}$ & $-2.497^{* * *}$ & $-2.500^{* * *}$ \\
\hline & {$[0.054]$} & {$[0.054]$} & {$[0.053]$} & {$[0.053]$} & {$[0.053]$} & {$[0.053]$} & {$[0.053]$} & {$[0.053]$} \\
\hline \multirow{2}{*}{ BookProb } & $5.032^{* * *}$ & $5.034^{* * *}$ & $5.032^{* * *}$ & $5.034^{* * *}$ & $5.039^{* * *}$ & $5.058^{* * *}$ & $5.052^{\star * *}$ & $5.056^{* * *}$ \\
\hline & {$[0.129]$} & {$[0.129]$} & {$[0.128]$} & {$[0.128]$} & {$[0.128]$} & {$[0.127]$} & {$[0.126]$} & {$[0.126]$} \\
\hline \multirow{2}{*}{ Home } & 0.030 & 0.030 & 0.030 & 0.030 & 0.029 & 0.027 & 0.027 & 0.027 \\
\hline & {$[0.047]$} & {$[0.047]$} & {$[0.047]$} & {$[0.047]$} & {$[0.047]$} & {$[0.047]$} & {$[0.047]$} & {$[0.047]$} \\
\hline \multirow{2}{*}{ GhostGame } & $0.980^{* *}$ & $0.713^{* *}$ & $0.625^{\star *}$ & $0.629^{\star *}$ & 0.215 & 0.182 & 0.089 & 0.093 \\
\hline & {$[0.452]$} & {$[0.338]$} & {$[0.301]$} & {$[0.258]$} & {$[0.201]$} & {$[0.162]$} & {$[0.123]$} & {$[0.115]$} \\
\hline \multirow{2}{*}{ Home $x$ GhostGame } & $-2.463^{\star * *}$ & $-1.803^{\star * *}$ & $-1.756^{\star * *}$ & $-1.647^{\star * *}$ & $-0.723^{\star *}$ & -0.446 & -0.172 & -0.142 \\
\hline & {$[0.882]$} & {$[0.636]$} & {$[0.579]$} & {$[0.482]$} & {$[0.352]$} & {$[0.283]$} & {$[0.213]$} & {$[0.198]$} \\
\hline League Fixed Effects & Yes & Yes & Yes & Yes & Yes & Yes & Yes & Yes \\
\hline Model & Logit & Logit & Logit & Logit & Logit & Logit & Logit & Logit \\
\hline Observations & 16,572 & 16,606 & 16,628 & 16,666 & 16,772 & 16,894 & 17,236 & 17,352 \\
\hline$R$-Squared & 0.142 & 0.142 & 0.142 & 0.142 & 0.142 & 0.143 & 0.142 & 0.142 \\
\hline
\end{tabular}

\begin{tabular}{|c|c|c|c|c|c|c|c|c|}
\hline \multirow{4}{*}{ ExchangeProb } & $-2.434^{\star * *}$ & $-2.434^{* * *}$ & $-2.433^{\star * *}$ & $-2.435^{\star * *}$ & $-2.434^{\star * *}$ & $-2.441^{\star * *}$ & $-2.438^{* * *}$ & $-2.440^{* * *}$ \\
\hline & {$[0.052]$} & {$[0.052]$} & {$[0.052]$} & {$[0.052]$} & {$[0.052]$} & {$[0.052]$} & {$[0.051]$} & {$[0.051]$} \\
\hline & $4.848^{\star * *}$ & $4.850^{\star * *}$ & $4.847^{\star * *}$ & $4.849^{\star * *}$ & $4.854^{* * *}$ & $4.869^{* * *}$ & $4.855^{\star * *}$ & $4.862^{* * *}$ \\
\hline & {$[0.123]$} & {$[0.122]$} & {$[0.122]$} & {$[0.122]$} & {$[0.122]$} & {$[0.121]$} & {$[0.120]$} & {$[0.120]$} \\
\hline \multirow{2}{*}{ Home } & 0.039 & 0.039 & 0.039 & 0.039 & 0.038 & 0.036 & 0.038 & 0.037 \\
\hline & {$[0.047]$} & {$[0.047]$} & {$[0.047]$} & {$[0.047]$} & {$[0.047]$} & {$[0.047]$} & {$[0.047]$} & {$[0.047]$} \\
\hline \multirow{2}{*}{ GhostGame } & $1.016^{\star *}$ & $0.755^{\star *}$ & $0.654^{\star *}$ & $0.658^{\star *}$ & 0.225 & 0.185 & 0.097 & 0.099 \\
\hline & {$[0.454]$} & {$[0.341]$} & {$[0.304]$} & {$[0.262]$} & {$[0.204]$} & {$[0.164]$} & {$[0.126]$} & {$[0.117]$} \\
\hline \multirow{2}{*}{ Home $x$ GhostGame } & $-2.527^{\star * *}$ & $-1.880^{\star * *}$ & $-1.816^{\star * *}$ & $-1.700^{* * *}$ & $-0.737^{\star *}$ & -0.442 & -0.192 & -0.165 \\
\hline & {$[0.884]$} & {$[0.636]$} & {$[0.584]$} & [0.487] & {$[0.355]$} & {$[0.286]$} & {$[0.216]$} & {$[0.201]$} \\
\hline League Fixed Effects & Yes & Yes & Yes & Yes & Yes & Yes & Yes & Yes \\
\hline Model & Logit & Logit & Logit & Logit & Logit & Logit & Logit & Logit \\
\hline Observations & 16,572 & 16,606 & 16,628 & 16,666 & 16,772 & 16,894 & 17,236 & 17,352 \\
\hline$R$-Squared & 0.144 & 0.144 & 0.144 & 0.144 & 0.144 & 0.145 & 0.144 & 0.145 \\
\hline
\end{tabular}

Notes: The dependent variable is the outcome of the game from the perspective of the respective team (home or away), equaling 1 for a win and 0 otherwise. ${ }^{*}$ ${ }^{* *}$, and ${ }^{* * *}$ indicate statistical significance at the $10 \%, 5 \%$, and $1 \%$ significance levels, respectively. SEs are reported in brackets and clustered at the game level. The underlying samples include observations from the 2014/2015 season until specified in I to VIII. BookProb denotes the average book probability across the six bookmakers adjusted for the overround. ExchangeProb denotes the adjusted average implied winning probability across the two betting exchanges.

The results from our analysis suggest that both bookmakers and bettors temporarily overestimate the winning probability of home teams during ghost games. Thus, we exploit whether betting on away teams yields a better return than betting on home teams. We analyze the wagering of an equal 1 currency unit per bet posted by the bookmaker Bet365 during the COVID-19-induced ghost games for two samples: ghost games played in all four major European leagues and ghost games played in the Bundesliga only. ${ }^{18}$ To this end, we calculate the cumulative payoff since the resumption of league operations in the Bundesliga (May 16, 2020). The results are displayed in Figure 2 for the whole sample and Figure 3 for the German Bundesliga only. The y-axis corresponds to the net payoff in the currency unit when wagering 1 currency unit per game, and the $\mathrm{x}$-axis corresponds to the number of days since resumption in the German Bundesliga. ${ }^{19}$

The results from Figure 2 suggest that betting on away teams during ghost games was highly profitable at the beginning but became negative after June 19, 2020. This is not unexpected given that by June 19, 2020, three of the four major leagues 
Table 3. Persistence Over Time

\begin{tabular}{|c|c|c|}
\hline & \multicolumn{2}{|c|}{ Outcome } \\
\hline & $\begin{array}{c}\text { I } \\
\text { Bookmaker }\end{array}$ & $\begin{array}{c}\text { II } \\
\text { Betting Exchange }\end{array}$ \\
\hline \multirow{2}{*}{ Constant } & $-2.496^{* * *}$ & $-2.437^{\star \star \star}$ \\
\hline & {$[0.053]$} & {$[0.051]$} \\
\hline \multirow{2}{*}{ BookProb } & $5.059^{* * *}$ & - \\
\hline & {$[0.126]$} & - \\
\hline \multirow{2}{*}{ ExchangeProb } & - & $4.865^{\star \star \star}$ \\
\hline & - & {$[0.120]$} \\
\hline \multirow{2}{*}{ Home } & 0.027 & 0.037 \\
\hline & {$[0.047]$} & {$[0.047]$} \\
\hline \multirow{2}{*}{ GhostGame } & $0.496^{*}$ & $0.530^{*}$ \\
\hline & {$[0.292]$} & {$[0.295]$} \\
\hline \multirow{2}{*}{ Time } & -0.062 & -0.066 \\
\hline & {$[0.043]$} & {$[0.043]$} \\
\hline \multirow{2}{*}{ Home x GhostGame } & $-1.356^{* * *}$ & $-1.389^{* * *}$ \\
\hline & {$[0.500]$} & {$[0.503]$} \\
\hline \multirow{2}{*}{ Home $x$ Time } & $0.184^{\star \star}$ & $0.185^{\star \star}$ \\
\hline & {$[0.072]$} & {$[0.073]$} \\
\hline League Fixed Effects & Yes & Yes \\
\hline Model & Logit & Logit \\
\hline Observations & 17,352 & 17,352 \\
\hline$R$-Squared & 0.143 & 0.145 \\
\hline
\end{tabular}

Notes: The dependent variable is the outcome of the game from the perspective of the respective team (home or away), equaling 1 for a win and 0 otherwise. ${ }^{*}, * *$, and ${ }^{\star * \star}$ indicate statistical significance at the $10 \%, 5 \%$, and $1 \%$ significance levels, respectively. SEs are reported in brackets and clustered at the game level. BookProb denotes the average book probability across the six bookmakers adjusted for the overround. ExchangeProb denotes the adjusted average implied winning probability across the two betting exchanges.

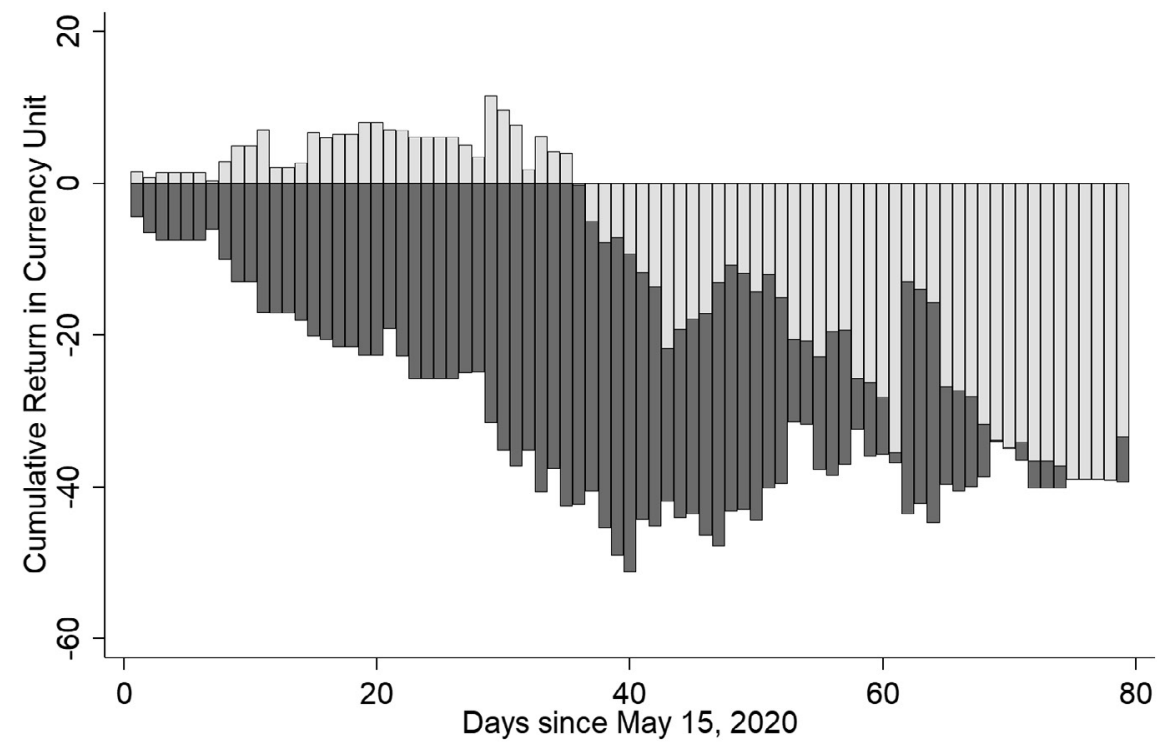

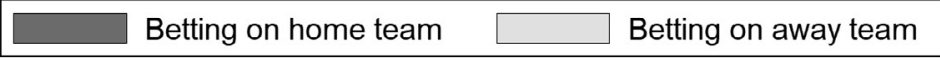

Figure 2. Return when betting on away and home teams (whole sample)

Notes: The figure displays the absolute cumulative return when wagering an equal 1 currency unit per bet. 
Table 4. League Difference

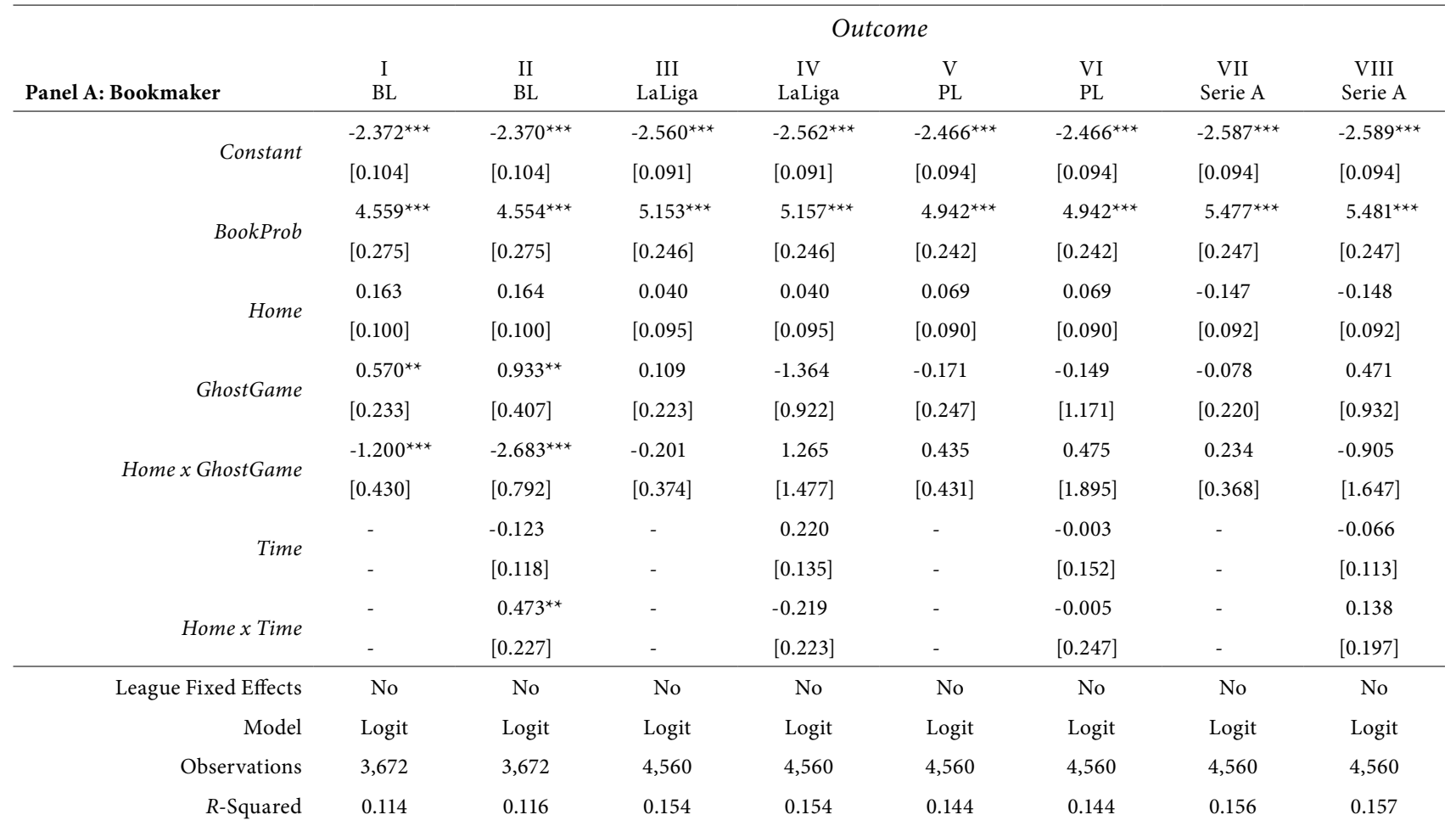

Panel B: Betting Exchange

\begin{tabular}{|c|c|c|c|c|c|c|c|c|}
\hline \multirow{4}{*}{ ExchangeProb } & $-2.300^{* * *}$ & $-2.299^{* * *}$ & $-2.484^{* * *}$ & $-2.485^{* * *}$ & $-2.420^{* * *}$ & $-2.420^{* * *}$ & $-2.526^{\star * *}$ & $-2.527^{\star * *}$ \\
\hline & {$[0.100]$} & {$[0.100]$} & [0.088] & [0.088] & [0.090] & [0.090] & [0.092] & [0.092] \\
\hline & $4.314^{\star * *}$ & $4.312^{* * *}$ & $4.952^{* * *}$ & $4.955^{\star * *}$ & $4.793^{\star * *}$ & $4.793^{\star * *}$ & $5.285^{\star * *}$ & $5.288^{* * *}$ \\
\hline & {$[0.260]$} & {$[0.260]$} & {$[0.234]$} & {$[0.234]$} & {$[0.231]$} & {$[0.231]$} & {$[0.235]$} & {$[0.235]$} \\
\hline \multirow{6}{*}{ Home $x$ GhostGame } & $0.189^{*}$ & $0.190^{*}$ & 0.033 & 0.032 & 0.079 & 0.079 & -0.133 & -0.134 \\
\hline & {$[0.099]$} & {$[0.099]$} & {$[0.094]$} & {$[0.094]$} & {$[0.090]$} & {$[0.090]$} & {$[0.091]$} & {$[0.091]$} \\
\hline & $0.577^{\star *}$ & $0.980^{\star *}$ & 0.132 & -1.270 & -0.166 & -0.169 & -0.090 & 0.496 \\
\hline & {$[0.235]$} & {$[0.408]$} & {$[0.229]$} & {$[0.940]$} & {$[0.250]$} & {$[1.171]$} & {$[0.223]$} & {$[0.940]$} \\
\hline & $-1.207^{* * *}$ & $-2.784^{* * *}$ & -0.253 & 1.184 & 0.419 & 0.498 & 0.227 & -0.799 \\
\hline & {$[0.432]$} & {$[0.795]$} & {$[0.381]$} & {$[1.505]$} & {$[0.438]$} & {$[1.902]$} & {$[0.371]$} & {$[1.661]$} \\
\hline \multirow{2}{*}{ Time } & - & -0.138 & - & 0.210 & - & 0.000 & - & -0.071 \\
\hline & - & {$[0.118]$} & - & [0.139] & - & {$[0.151]$} & - & {$[0.114]$} \\
\hline \multirow{2}{*}{ Home $x$ Time } & - & $0.504^{\star *}$ & - & -0.215 & - & -0.011 & - & 0.124 \\
\hline & - & {$[0.226]$} & - & {$[0.228]$} & - & {$[0.246]$} & - & [0.199] \\
\hline League Fixed Effects & No & No & No & No & No & No & No & No \\
\hline Model & Logit & Logit & Logit & Logit & Logit & Logit & Logit & Logit \\
\hline Observations & 3,672 & 3,672 & 4,560 & 4,560 & 4,560 & 4,560 & 4,560 & 4,560 \\
\hline$R$-Squared & 0.114 & 0.115 & 0.156 & 0.156 & 0.147 & 0.147 & 0.160 & 0.160 \\
\hline
\end{tabular}

Notes: The dependent variable is the outcome of the game from the perspective of the respective team (home or away), equaling 1 for a win and 0 otherwise. ${ }^{*}$ ${ }^{* *}$, and ${ }^{* * *}$ indicate statistical significance at the $10 \%, 5 \%$, and $1 \%$ significance levels, respectively. SEs are reported in brackets and clustered at the game level. BookProb denotes the average book probability across the six bookmakers adjusted for the overround. ExchangeProb denotes the adjusted average implied winning probability across the two betting exchanges. BL and PL stand for the German Bundesliga and English Premier League, respectively. LaLiga denotes the first division in Spain and Serie A the first division in Italy. 


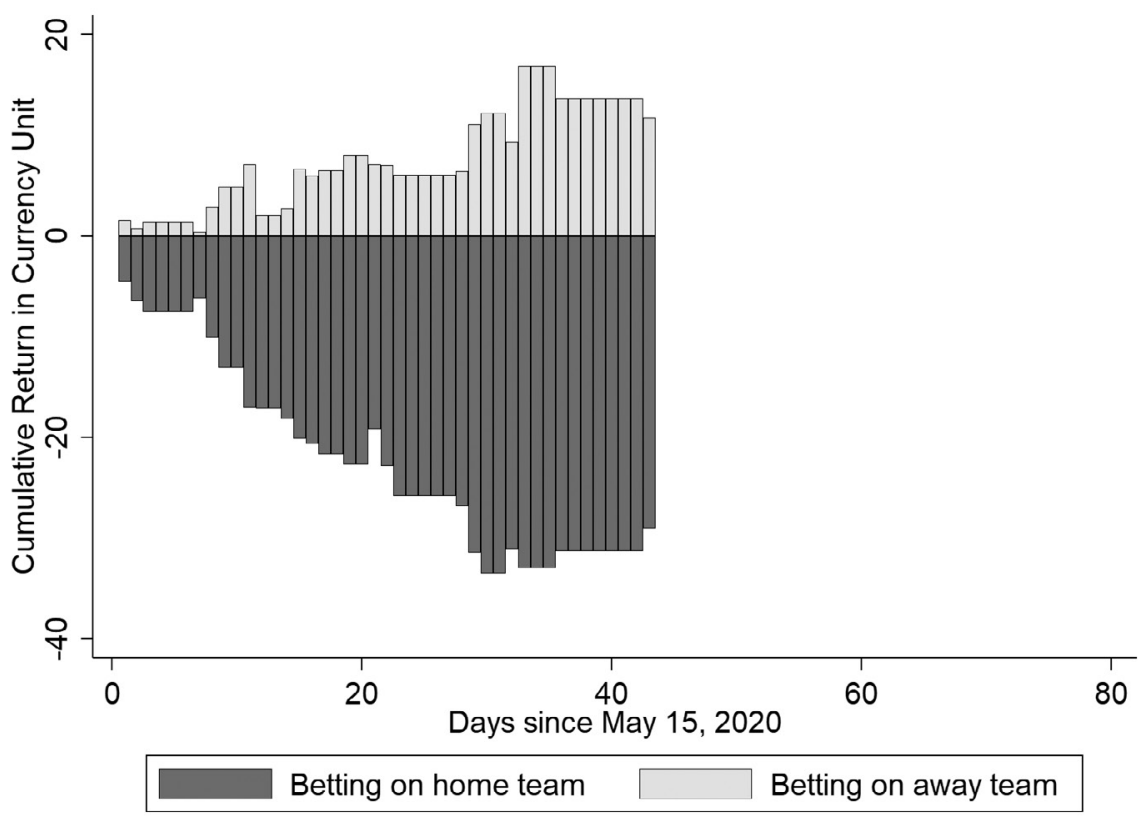

Figure 3. Return when betting on away and home Teams (Bundesliga only)

Notes: The figure displays the absolute cumulative return when wagering an equal 1 currency unit per bet.

had already resumed operations. Betting on away teams remained negative until the end of the season and gradually converged with the cumulative, negative return from betting on home teams. The positive returns are particularly driven by the German Bundesliga, as Figure 3 suggests. A similar betting strategy for ghost games in the German Bundesliga would have yielded a positive cumulative net payoff until the end of the 2019/2020 season (June 27,2020), equaling a total return of over $14.4 \%$, while betting on the home team would have yielded a return of $-35.4 \% .{ }^{20}$

Thus, betting on the away team not only yielded a better return than betting at random, the expected return of which would be negative due to the overround of bookmakers, it also yielded a positive profit-at least for the early period of ghost games. This finding implies that betting markets are not semi-strong form efficient since it should not be possible to achieve above-average returns (Vaughan Williams, 2005).

\section{Discussion and Conclusion}

We find robust evidence that betting markets are inefficient, at least in semi-strong form: Bookmakers and bettors alike systematically overestimated (underestimated) the home (away) team's winning probability during the early stages of the post-resumption period, suggesting that the new information of a reduced home advantage in ghost games was not fully incorporated in the betting odds at that point, and only after approximately five weeks later correctly reflected the winning probabilities. The effect is particularly driven by the German Bundesliga, which resumed operations as the first major professional football league after the lockdown. A simple betting strategy requiring an equal bet on away teams would have yielded a large positive return for the first weeks after resumption of the German Bundesliga.

These findings are in contrast to contemporaneous work by Hegarty (2021), who found an improvement in market predictions in ghost games utilizing the top four professional European football leagues in the 2019/2020 and 2020/2021 seasons. A potential reason behind this contrasting finding is that he used a different approach. In particular, he used the Asian handicap market, the actual goal difference of a game as the outcome variable and compared 754 ghost games played in the 2019/2020 and 2020/2021 seasons to an equal set of regular games immediately prior to the change. Moreover, the author did not specifically examine an adaption process of bookmakers over time. If we analyze the whole time period in Table 2, Column VIII, we also find no evidence of inefficiency. However, this clouds the fact that inefficiency is present in the first five weeks after resumption and vanishes thereafter. 
In further contemporaneous works, Winkelmann et al. (2020a) and Fischer and Haucap (2020a) analyzed bookmakers' reactions to the reduction in the home advantage in Germany. In deviation from our results, Winkelmann et al. (2020a) concluded that mispricing persisted throughout ghost games in the German Bundesliga. One reason for this deviation might be that Winkelmann et al. (2021) utilized a different specification that mainly captures the average effect of playing at home or away in ghost games. Fischer and Haucap (2020a) suggested a slow to non-existing adaption process in the German professional leagues.

There exist several potential mechanisms that could lead to temporal inefficiency in betting markets. For example, bookmakers might be well aware of the decreased home advantage and the change in the winning probabilities. Nevertheless, they intentionally decided not to adjust the odds to exploit bettor preferences and maximize their profits (Levitt, 2004). However, given our evidence that betting exchange odds exhibit a very similar bias, such an explanation seems unlikely. It rather suggests that both bettors and bookmakers failed to update their predications, at least in the beginning of the ghost game period. However, it remains unclear whether market participants truly updated their beliefs or if the home advantage gradually returned to its pre-pandemic level, and thus, the inefficiency in betting odds vanished. For instance, Fischer and Haucap (2020b) argued that players might become accustomed to the new situation without spectators, resulting in the ghost game effect gradually disappearing. Singleton et al. (2021) proposed an additional explanation, arguing that potentially bettors and bookmakers may have viewed the effect of ghost games on home advantage in the German Bundesliga as a plausible outlier.

Evidence suggests that the home advantage is, on average, reduced during ghost games (Cueva, 2020; Scoppa, 2021), although the extent varies across the leagues (Bryson et al., 2021). This also holds for the four major European leagues that are utilized in our study. Bryson et al. (2021, p. 3) illustrated evidence of a decreased home advantage in the German Bundesliga, Italian Serie A, Spanish La Liga, and English Premier League both in terms of goal difference and yellow cards. The fact that we observe only temporal market inefficiency in the first league to resume operations, the German Bundesliga, thus supports the view that market participants have failed to update their beliefs immediately but did so following the resumption of the other European leagues. If market participants had not adapted, we would expect to see similar evidence of inefficiency in one of the other European leagues. Nonetheless, we cannot rule out the possibility that these alternative explanations, at least partially, also contributed to the results.

While one advantage of our study is the clean arrival of new public information due to the COVID-19-induced ghost games, there were additional temporal changes in football rules that might have impacted the dynamics of the game. For example, to prevent injuries and excessive strain, the International Football Association Board (IFAB) introduced the possibility of five substitutions, instead of three, during tightly scheduled ghost games. However, because both home and away teams are affected by these changes, they should not systematically influence the home advantage (Scoppa, 2021). Furthermore, the announcement of ghost games may also have led to changes in the betting market. For example, the characteristics of bettors or the bettors' attention to football games could have shifted. However, the effects of these changes not included in the implied probabilities are captured by the dummy variable GhostGame and are thus not expected to alter our main conclusions.

Similar to Choi and Hui (2014), our inferences are generalizable to financial markets only insofar as the behavior of bettors is comparable to investors in traditional financial markets. Notably, market participants and incentives may be different from those of traditional financial markets. Thus, we can provide only limited evidence for traditional financial markets. However, our results suggest that individuals may not immediately adjust their beliefs rationally following the arrival of new public information. This may partially explain why individuals underreact to new public information, a finding that has also been documented in the traditional financial market (Cohen et al., 2002). The implications for sports betting markets are more straightforward: The fact that these markets are, at most, weak-form efficient indicates that they provide ample opportunities for bettors to earn abnormal returns by quickly reacting to surprise announcements and disclosures.

For further research, it might be promising to examine home advantage and the question of whether bookmakers and bettors correctly assess the winning probability of a game with full, partial, or no crowd. At the start of the 2020/2021 season, European football clubs were allowed to let relatively few spectators enter the stadiums in compliance with protection concepts. ${ }^{21}$ Due to the fluctuations in the infection figures, there were changing regulations across and within European 
football leagues. ${ }^{22}$ Some games in the Champions and Europa League even had to be played in neutral venues due to travel restrictions. These avenues might be valuable for studying the efficiency of betting markets.

\section{Endnotes}

1 The authors received no financial support for the research, authorship, and/or publication of this article.

2 We excluded France, as the French League 1 schedule was terminated without ghost games being played.

3 Legaz-Arrese et al. (2013) provided an overview of home advantage in different team sports.

4 On the one hand, the crowd tends to stimulate players' effort, motivation, and energy; on the other hand, it might subconsciously influence the referee to favor the home team as an indirect effect (Van Damme \& Baert, 2019).

5 Poli et al. (2020) provided a simple overview of the evolution of the home advantage by comparing the share of home wins before and during the pandemic for leagues with at least 40 games played after the season resumed. While the top Greek division showed the greatest decrease in the percentage of home wins (-15.1\%), some leagues (21 out of 63) exhibited an increase in home wins, with Switzerland being the country where home teams have won the most compared to their pre-pandemic record $(+8.5 \%)$.

6 See, for example, the following articles:

https://theconversation.com/as-football-returns-in-empty-stadiums-four-graphs-show-how-home-advantage-disappears-138685 (Singleton et al., 2020); https://www.befootball.com/new/covid-19-takes-away-home-advantage-836200 (BeSoccer, 2020); https://www. dw.com/en/bundesliga-ghost-games-have-killed-home-advantage/a-53634123 (Da Silva, 2020).

7 The probability of a home team win is derived as follows: $\hat{p}_{h}=\frac{1 / O_{h}}{1 / O_{h}+1 / O_{d}+1 / O_{a}}$.

There exist also other approaches to adjust the bookmaker's implied probabilities (see Clarke et al., 2017 for an overview).

8 For some games in the earlier seasons, odds from either Betfair or Matchbook are missing. However, we have at least one odd from a betting exchange for all games.

9 We also check a random assignment of games to either home or away and find that the estimates remain robust. We reach similar conclusions when examining two separate models for home and away teams.

10 The results remain virtually the same when we exclude league fixed effects.

11 The German Bundesliga resumed operations on May 16, 2020. Thus, we calculate Time as the difference between the game date and May 15, 2020, in weeks for games after the resumption. Thus, Time takes on a positive value for all games after May 15, 2020 and zero for all games prior to this date. We also tested the number of days or the number of ghost games played since resumption as alternative specifications and reach very similar conclusions.

12 Utilizing other end dates does not alter our conclusions since the average effect vanishes after June 21, 2020.

13 The results remain virtually the same when we utilize the odds quoted at either of the two betting exchanges.

14 Thus, we are not able to confirm the findings of some prior studies (Vlastakis et al., 2009; Winkelmann et al., 2020), suggesting a home bias in bookmaker's odds. For an overview, see Winkelmann et al. (2020), who provided a comprehensive comparison of studies that examine biases in betting odds.

15 Adding more covariates such as team strength to our model should not alter our main results because each team alternatively takes the role of the home team and away team. Indeed, our results remain virtually identical if we control for team strength measured as the difference in market values of the two teams derived from www.transfermarkt.com.

16 The results also remain virtually the same when we do not correct for the overround of bookmakers.

17 The results remain virtually the same when we use league specific resumption dates to calculate the time variable (both in weeks and days) instead of utilizing time as defined by the difference in weeks from the ghost game to the resumption date from the German Bundesliga.

18 We also check the results using the odds from the other five bookmakers and find virtually no difference. We derive similar conclusions when utilizing odds from the betting exchange.

19 If no ghost game took place on a certain day, the cumulative return of the last game day is shown.

20 At the peak of this strategy in terms of return on May 26, the strategy of betting on the away team would have yielded a return of over $32.2 \%$ and $-77.5 \%$ when betting solely on home teams.

21 For instance, in the 2018/2019 season, Borussia Dortmund had an average of over 80,000 spectators per game in their home venue. As of beginning of April 2021, the club had only played three games with spectators present at Signal-Iduna Park, but only in front of approximately 7,000 spectators per game (Kicker, 2021).

22 For example, in Germany, from November 2, spectators in German stadia were again prohibited due to rising case numbers. In England, the British Health Minister Matt Hancock declared on December 14 that the capital would be grouped into the highest COVID-19 warning level due to the drastically increasing number of cases and therefore did not allow any fans into the stadiums. At that time, Liverpool FC, Everton FC, Southampton FC, and Brighton \& Hove Albion were the only teams allowed to play in front of a limited number of spectators in their own stadium (Sportschau, 2020). 


\section{References}

Angelini, G., \& De Angelis, L. (2019). Efficiency of online football betting markets. International Journal of Forecasting, 35(2), $712-721$

Benz, L. S., \& Lopez, M. J. (2020). Estimating the change in soccer's home advantage during the COVID-19 pandemic using bivariate Poisson regression. ArXiv Preprint ArXiv:2012.14949.

Berkowitz, J. P., Depken II, C. A., \& Gandar, J. M. (2017). A favorite-longshot bias in fixed-odds betting markets: Evidence from college basketball and college football. The Quarterly Review of Economics and Finance, 63, 233-239.

Bernardo, G., Ruberti, M., \& Verona, R. (2019). Semi-strong inefficiency in the fixed odds betting market: Underestimating the positive impact of head coach replacement in the main European soccer leagues. The Quarterly Review of Economics and Finance, $71,239-246$.

BeSoccer. (2020, May 19). COVID-19 takes away home advantage. https://www.besoccer.com/new/covid-19-takes-away-home-advantage- 836200

Bizzozero, P., Flepp, R., \& Franck, E. (2016). The importance of suspense and surprise in entertainment demand: Evidence from Wimbledon. Journal of Economic Behavior \& Organization, 130, 47-63.

Bizzozero, P., Flepp, R., \& Franck, E. (2018). The effect of fast trading on price discovery and efficiency: Evidence from a betting exchange. Journal of Economic Behavior \& Organization, 156, 126-143.

Borghesi, R. (2014). The impact of the disposition effect on asset prices: Insight from the NBA. Journal of Economics and Finance, 38(4), 698-711.

Brown, A., Rambaccussing, D., Reade, J. J., \& Rossi, G. (2018). Forecasting with social media: Evidence from tweets on soccer matches. Economic Inquiry, 56(3), 1748-1763.

Bryson, A., Dolton, P., Reade, J. J., Schreyer, D., \& Singleton, C. (2021). Causal effects of an absent crowd on performances and refereeing decisions during Covid-19. Economics Letters, 198, 109664.

Cain, M., Law, D., \& Peel, D. (2000). The favourite-longshot bias and market efficiency in UK football betting. Scottish Journal of Political Economy, 47(1), 25-36.

Choi, D., \& Hui, S. K. (2014). The role of surprise: Understanding overreaction and underreaction to unanticipated events using inplay soccer betting market. Journal of Economic Behavior \& Organization, 107, 614-629.

Clarke, S., Kovalchik, S., \& Ingram, M. (2017). Adjusting bookmaker's odds to allow for overround. American Journal of Sports Science, 5(6), 45.

Cohen, R. B., Gompers, P. A., \& Vuolteenaho, T. (2002). Who underreacts to cash-flow news? Evidence from trading between individuals and institutions. Journal of Financial Economics, 66(2/3), 409-462.

Courneya, K. S., \& Carron, A. V. (1992). The home advantage in sport competitions: A literature review. Journal of Sport and Exercise Psychology, 14(1), 13-27.

Croxson, K., \& Reade, J. J. (2014). Information and efficiency: Goal arrival in soccer betting. The Economic Journal, 124(575), 62-91.

Cueva, C. (2020). Animal spirits in the beautiful game: Testing social pressure in professional football during the COVID-19 lockdown. Working Paper.

Da Silva, M. (2020, May 30). Bundesliga: 'Ghost games' have killed home advantage. Deutsche Welle. https://www.dw.com/en/bundesliga-ghost-games-have-killed-home-advantage/a-53634123

Deutscher, C., Frick, B., \& Ötting, M. (2018). Betting market inefficiencies are short-lived in German professional football. Applied Economics, 50(30), 3240-3246.

Dilger, A., \& Vischer, L. (2020). No home bias in ghost games. Diskussionspapier des Instituts für Organisationsökonomik, No. 7/2020, Westfälische WilhelmsUniversität Münster, Institut für Organisationsökonomik, Münster. https://www.econstor.eu/bitstre am/10419/223208/1/1727445767.pdf

Dixon, M. J., \& Coles, S. G. (1997). Modelling association football scores and inefficiencies in the football betting market. Journal of the Royal Statistical Society: Series C (Applied Statistics), 46(2), 265-280.

Dixon, M. J., \& Pope, P. F. (2004). The value of statistical forecasts in the UK association football betting market. International Journal of Forecasting, 20(4), 697-711.

Edelman, D. (2002). A competitive horse-race handicapping algorithm based on analysis of covariance. In L. Vaughan-Williams (Ed.), The economics of gambling (pp. 106-113). Routledge.

Elaad, G., Reade, J. J., \& Singleton, C. (2020). Information, prices and efficiency in an online betting market. Finance Research Letters, 35, 101291.

Endrich, M., \& Gesche, T. (2020). Home-bias in referee decisions: Evidence from 'ghost matches' during the COVID-19 pandemic. Center for Law \& Economics Working Paper Series.

Fama, E. F. (1970). Efficient capital markets: A review of theory and empirical work. The Journal of Finance, 25(2), 383-417.

Fédération Internationale de Football Association (FIFA). (2011, March 26). Sports betting under the microscope. https://www.fifa. com/who-we-are/news/sports-betting-under-the-microscope-1406983

Ferraresi, M., \& Gucciardi, G. (2020). Team performance and audience: experimental evidence from the football sector. Working Paper No. 760, Società Italiana di Economia Pubblica. https://www.researchgate.net/profile/Massimiliano-Ferraresi/publication/344106744_Team_performance_and_audience_experimental_evidence_from_the_football_sector/links/5f526a86299bf13a319f78ce/Team-performance-and-audience-experimental-evidence-from-the-football-sector.pdf

Fischer, K., \& Haucap, J. (2020a). Betting market efficiency in the presence of unfamiliar shocks: The case of ghost games during the COVID-19 pandemic. CESifo Working Paper No. 8526.

Fischer, K., \& Haucap, J. (2020b). Does crowd support drive the home advantage in professional soccer? Evidence from German ghost games during the COVID-19 pandemic. DICE Discussion Paper No. 344.

Flepp, R., Nüesch, S., \& Franck, E. (2017). The liquidity advantage of the quote-driven market: Evidence from the betting industry. The Quarterly Review of Economics and Finance, 64, 306-317. 
Forrest, D., \& Simmons, R. (2000). Forecasting sport: The behaviour and performance of football tipsters. International Journal of Forecasting, 16(3), 317-331.

Forrest, D., \& Simmons, R. (2008). Sentiment in the betting market on Spanish football. Applied Economics, 40(1), 119-126.

Franck, E., Verbeek, E., \& Nüesch, S. (2010). Prediction accuracy of different market structures: Bookmakers versus a betting exchange. International Journal of Forecasting, 26(3), 448-459.

Franke, M. (2020). Do market participants misprice lottery-type assets? Evidence from the European soccer betting market. The Quarterly Review of Economics and Finance, 75, 1-18.

Goddard, J., \& Asimakopoulos, I. (2004). Forecasting football results and the efficiency of fixed-odds betting. Journal of Forecasting, 23(1), 51-66.

Goumas, C. (2014). How does crowd support contribute to home advantage in soccer? Journal of Sport Behavior, $37(3), 236$.

Hausch, D. B., Ziemba, W. T., \& Rubinstein, M. (1981). Efficiency of the market for racetrack betting. Management Science, 27(12), $1435-1452$.

Hegarty, T. (2021). Information and price efficiency in the absence of home crowd advantage. Applied Economics Letters. https://doi. org/10.1080/13504851.2021.1883525

Hvattum, L. M. (2013). Analyzing information efficiency in the betting market for association football league winners. The Journal of Prediction Markets, 7(2), 55-70.

Kicker. (2021, April 5). Zuschauer/Bundesliga 2020/21. https://www.kicker.de/bundesliga/zuschauer/2020-21

Konaka, E. (2021). Home advantage of European major football leagues under COVID-19 pandemic. ArXiv Preprint ArXiv:2101.00457.

Kuypers, T. (2000). Information and efficiency: An empirical study of a fixed odds betting market. Applied Economics, 32(11), $1353-1363$.

Legaz-Arrese, A., Moliner-Urdiales, D., \& Munguía-Izquierdo, D. (2013). Home advantage and sports performance: Evidence, causes and psychological implications. Universitas Psychologica, 12(3), 933-943.

Levitt, S. D. (2004). Why are gambling markets organised so differently from financial markets? The Economic Journal, 114(495), $223-246$

Marshall, B. R. (2009). How quickly is temporary market inefficiency removed? The Quarterly Review of Economics and Finance, 49(3), 917-930.

McCarrick, D., Bilalic, M., Neave, N., \& Wolfson, S. (2021). Home advantage during the COVID-19 pandemic: Analyses of European football leagues. Psychology of Sport and Exercise, 102013.

Nevill, A. M., Newell, S. M., \& Gale, S. (1996). Factors associated with home advantage in English and Scottish soccer matches. Journal of Sports Sciences, 14(2), 181-186.

Pettersson-Lidbom, P., \& Priks, M. (2010). Behavior under social pressure: Empty Italian stadiums and referee bias. Economics Letters, 108(2), 212-214.

Poli, R., Besson, R., Ravenel, L., \& Gonzalez, T. (2020). What about the home advantage after the COVID-19 pandemic? CIES Football Observatory. https://football-observatory.com/IMG/sites/b5wp/2020/wp304/en/

Pollard, R. (2006). Home advantage in soccer: Variations in its magnitude and a literature review of the inter-related factors associated with its existence. Journal of Sport Behavior, 29(2), 169.

Ponzo, M., \& Scoppa, V. (2018). Does the home advantage depend on crowd support? Evidence from same-stadium derbies. Journal of Sports Economics, 19(4), 562-582.

Reade, J., \& Singleton, C. (2020). European football after COVID-19. In M. Billio \& S. Varotto (Eds.), A new world post COVID-19: Lessons for business, the finance industry and policy makers (pp. 348-358). Ca' Foscari University Press.

Rue, H., \& Salvesen, O. (2000). Prediction and retrospective analysis of soccer matches in a league. Journal of the Royal Statistical Society: Series D (the Statistician), 49(3), 399-418.

Scoppa, V. (2021). Social pressure in the stadiums: Do agents change behavior without crowd support? Journal of Economic Psychology, 82, 102344.

Singleton, C., Bryson, A., Dolton, P., Reade, J., \& Schreyer, D. (2021). What can we learn about economics from sport during COVID-19? https://dx.doi.org/10.2139/ssrn.3770193

Singleton, C., Schreyer, D., \& Reade, J. (2020, May 15). As football returns in empty stadiums, four graphs show how home advantage disappears. The Conversation. https://theconversation.com/as-football-returns-in-empty-stadiums-four-graphs-show-how-homeadvantage-disappears- 138685

Smith, M. A. (2002). The impact of tipster information on bookmakers' prices in UK horse-race markets. In L. Vaughan-Williams (Ed.), The economics of gambling (pp. 67-78). Routledge.

Sors, F., Grassi, M., Agostini, T., \& Murgia, M. (2020). The sound of silence in association football: Home advantage and referee bias decrease in matches played without spectators. European Journal of Sport Science, 1-9.

Sportschau. (2020, December 14). Premier league: Keine zuschauer mehr bei spielen in London. https://www.sportschau.de/fussball/ mehr-internationale-ligen/premier-league-ohne-zuschauer-108.html

Sung, M. C., Johnson, J. E. V., \& Bruce, A. C. (2005). Searching for semi-strong form inefficiency in the UK racetrack betting market. In L. Vaughan Williams (Ed.), Information efficiency in financial and betting markets (pp. 179-192). Cambridge University Press.

Tilp, M., \& Thaller, S. (2020). COVID-19 has turned home-advantage into home-disadvantage in the German Soccer Bundesliga. Frontiers in Sports and Active Living, 2, 165.

Van Damme, N., \& Baert, S. (2019). Home advantage in European international soccer: Which dimension of distance matters? Economics: The Open-Access, Open-Assessment E-Journal, 13(2019-50): 1-17.

Vaughan Williams, L. (2005). Information efficiency in financial and betting markets. Cambridge University Press.

Vlastakis, N., Dotsis, G., \& Markellos, R. N. (2009). How efficient is the European football betting market? Evidence from arbitrage and trading strategies. Journal of Forecasting, 28(5), 426-444. 
Winkelmann, D., Deutscher, C., \& Ötting, M. (2021). Bookmakers' mispricing of the disappeared home advantage in the German Bundesliga after the COVID-19 break. Applied Economics, 53(28), 3054-3064.

Winkelmann, D., Ötting, M., \& Deutscher, C. (2020). Betting market inefficiencies in European football: Bookmakers' mispricing or pure chance? Bielefeld Working Papers in Economics and Management No. 06-2020. 